Original scientific paper

\title{
A SIMULATION METHOD FOR RAIL TRANSIT SIGN OPTIMIZATION
}

\author{
Ouyang, K. M. ${ }^{*} \&$ Liu, S. F. ${ }^{* *}$ \\ * Beijing Jingtou Urban Utility Tunnel Investment Co., Ltd., Beijing, China \\ ** School of Economics and Management, Beijing Jiaotong University, Beijing, China \\ E-Mail: ouyang6114@163.com, shfliu@ bjtu.edu.cn
}

\begin{abstract}
The guidance signs in rail transit stations directly affect the smoothness and effectiveness of passengers' travel. The reasonable layout of guidance signs plays a role in the station system, which can reasonably guide passengers' walking routes in the station, reduce their walking time, realize effective traffic guidance in the station, and bring great convenience to passengers' travel. In this paper, the simulation selects Beijing rail transit Xizhimen station as an example for analysis, introduces the Xizhimen junction station, and analyses the passenger flow line of Xizhimen station. Based on this, the values of various parameters required for the practical application of the mathematical model were determined, so as to obtain a complete design model for the optimization of the layout of guide signs, and Anylogic software was chosen to solve the model. Finally, the optimized location of the optimized sign is restored to the actual situation of Xizhimen Station to verify the validity of the model.

(Received in June 2021, accepted in October 2021. This paper was with the authors 1 month for 1 revision.)
\end{abstract}

Key Words: Urban Rail Transit, Guidance Sign, Layout Optimization, Simulation

\section{INTRODUCTION}

The rail transit guidance sign is a kind of public facility identification symbol with orientation and guidance, which is responsible for allowing passengers to identify the best ride and transfer routes more quickly and travel safely in a certain underground space environment. However, rail transit signs in underground space do not meet the needs of all passengers well, and some of them may bring visual misleading to passengers and cause many unnecessary troubles. First of all, there is no ground building as a reference in the underground closed complex space environment. So passengers' information acceptance ability will be greatly reduced, and it is difficult for them to distinguish the direction quickly and accurately in the unfamiliar complex environment. Secondly, the setting of signs will also have a great impact on passengers' information acceptance ability. Too much information will lead to visual confusion, the signs that are not set up in a reasonable and standard way will affect passengers, or the content of the signs that cannot be easily understood by passengers will make passengers feel confused too. Then, the behaviour of passengers is also very uncertain, and many factors such as age, thinking style, whether they are local or not, whether they take rail transit for the first time, etc. will have an impact on the understanding of the content of guidance signs, which may cause many problems. Thus, the guidance signs in rail transit stations directly affect the smoothness and effectiveness of passengers' travel. The reasonable setting of guidance signs has a significant impact on passengers' safe and convenient travel in rail transit.

To ensure the smooth passage of passengers in the underground space of rail transit stations, reasonable guidance functions are required to help passengers reach their destinations, and rail transit guidance signs have gained attention in academic circles [1,2]. Rail transit guidance signs are usually designed to tell passengers where they are located, and passengers rely on the functional design of the sign system. When passengers cannot find the information they need, they will be annoyed and anxious and generate negative emotions [3, 4]. Some scholars have also studied the functional design of rail transit signs from a cultural perspective and found that 
the visual elements and wording used in the symbols can reveal not only linguistic differences, but also the differences in cultural norms and values [5].

The space planning of guidance signs is the focus of sign system design, and a series of factors should be considered in the process of setting rail transit signs. For example, the setting of sign location and form is the key to the success of rail transit research [6], and the location of guidance signs needs to grasp and analyse the specific content of the whole guiding system of rail transit station [7]. Secondly, a reasonable visual distance and the number of advance guidance signs help passengers to be able to find their destinations and entrances and exits smoothly in the station [8]. In addition, the scale, the shape, the use of standard terminology, the understand ability of vocabulary, the standardization of Chinese and English settings of rail transit guidance signs, etc. have important effects on the visibility and effectiveness of the signs [9].

The reasonable layout of rail transit signs is a good way to act the information of the external environment on pedestrian behaviour [10]. The amount of load information, the location of information display, the comprehensibility of content and many other aspects related to the content of signs [11-14]. In terms of sign location, relevant studies using path finding experiments to determine location can also be found [15]. In addition, reasonable pricing can improve the guiding range and service effectiveness $[16,17]$. Similarly, some scholars have also studied the scheme by algorithm design and simulation model [18, 19].

In this paper, the simulation selects Beijing rail transit Xizhimen station as an example for analysis, introduces the Xizhimen junction station, and analyses the passenger flow line of Xizhimen station. Based on this, the values of various parameters required for the practical application of the mathematical model were determined, so as to obtain a complete design model for the optimization of the layout of guide signs, and Anylogic software was chosen to solve the model. Finally, the optimized location of the optimized sign is restored to the actual situation of Xizhimen Station to verify the validity of the model.

\section{PROBLEM DESCRIPTION AND MODELLING}

\subsection{Problem description}

The space composition of guidance signs in urban rail transit station has been determined in the design phase and construction phase, and the nodes of alternative locations for passenger guidance sign placement can be determined according to the building node analysis. From these alternative locations, the most suitable layout location is selected according to certain objectives to meet the needs of passengers, i.e., $q$ alternative locations are selected from $m$ alternative locations in a station according to certain calculation to guide the passenger flow to achieve certain guidance purposes ( $q$ and $m$ represent the number of locations).

The alternative locations refer to the location points that may change the direction of passengers' way finding within the scope of guidance signs in rail transit stations. These points are the alternative points for setting guidance signs, such as entrances and exits, staircases, flow intersections, corners and other special locations with spatial characteristics to arrange the relevant guidance signs. One of the challenges that urban rail transit is facing is the congestion in the station during peak hours. In cities, commuting demand is an important demand met by urban rail transit, but its occurrence is relatively concentrated, thus leading to the problem of congestion in stations within a short period of time when a large number of commuting occurs simultaneously during peak periods. This model addresses this problem and seeks to make it possible for passengers to reach their destinations in the shortest time. In order to allow passengers to reach their destinations as soon as possible and reduce congestion, it is necessary to ensure that the flow chosen by each passenger is within a certain amount of traffic, and to set a reasonable number of guide signs so that passengers can choose the shortest route to pass. 
Thereby, the time spent by passengers in the station can be shortened and the number of passengers passing through the station at the same time can be reduced. Therefore, the optimization objective of this study is the shortest sum of the route length taken by all passengers.

\subsection{Model building}

In this study, the station is abstracted into a topological graph $G(V, E)$, where $V$ denotes the set of all nodes (including entrance/exit points, platforms and alternative points), $E$ denotes the set of all arcs, and $G(V, E)$ denotes the graph composed of all points and edges through certain connection relationships. In order to clarify the meaning of the model construction, the passenger flow lines in stations, the number of each demand and its related symbols are defined in this paper as follows:

(1) a total of $k$ demands, numbered $k=1,2, \ldots, K$, and the set of all demands is $K$.

(2) the size of the passenger flow line for the $k^{\text {th }}$ demand is $h^{k}$, the starting and ending point under the $k^{\text {th }}$ demand are $o^{k}$ and $d^{k}$, respectively, and $O(D)$ denotes the set of starting points (ending points) of all demands.

(3) $w^{k}$ represents the importance of demand $k$.

(4) $l_{i j}$ denotes the edge between nodes $i$ and $j$. Whether the passenger flow of the $k^{\text {th }}$ demand passes through $l_{i j}$ is denoted by $x_{i j}^{k}$.

(5) the length of each edge in the graph is $c_{i j}$, the maximum passenger flow line allowed to pass by the edge is $u_{i j}$, and the total number of markers placed at each node is less than $p$. According to the above description, the model built with the total path shortest as the objective function can be expressed as

$$
\min \sum_{k \in K} \sum_{i \in V} \sum_{j \in V} c_{i j} x_{i j}^{k} h^{k} w^{k}
$$

Subject to:

$$
\begin{gathered}
\sum_{j \in V} x_{o^{k} j}^{k}=1 \quad \forall k \in K \\
\sum_{i \in V} x_{i d^{k}}^{k}=1 \quad \forall k \in K \\
\sum_{j \in V / O} x_{i j}^{k}=\sum_{j \in V / D} x_{i j}^{k} \quad \forall i \in V /\{O \cup D\}, k \in K \\
\sum_{k \in K} x_{i j}^{k} h^{k} \leq u_{i j} \quad \forall i \in V, j \in V \\
f\left(x_{i j}^{k}\right) \leq n \quad \forall j \in V \\
x_{i j}^{k}=\{0,1\} \quad \forall k \in K, i \in V, j \in V
\end{gathered}
$$

where constraints (2) and (3) denote if and only if each demand has one origin and one endpoint, respectively; constraint (4) is a flow balance constraint, which indicates that each demand entering a point must come out from that point; constraint (5) is a flow constraint for each arc; and constraint (6) is a constraint on the amount of information placed at each alternative point. The function $f\left(x_{i j}^{k}\right)$ denotes the number of non-repeating endpoints of the demands passing through node $j$. Since the endpoints of different demands may be the same, a simple sum of the number of endpoints cannot be used to represent the amount of information that needs to be placed at point $j$. Let the set $D_{j}$ store all the endpoints of the demands passing through $j$ and calculate $x_{i j}^{k} d^{k}$. If $x_{i j}^{k} d^{k}$ is not equal to 0 , store it into the set $D_{j}$, remove the duplicate items, and get $D_{j}^{\prime}$. The number of elements in $D_{j}^{\prime}$ is $f\left(x_{i j}^{k}\right)$; constraint (7) indicates that $x_{i j}^{k}$ is a 0-1 variable. 


\section{ALGORITHM DESIGN AND VERIFICATION}

\subsection{Heuristic algorithm}

When constructing heuristic algorithm, calculate the shortest path for all passenger flows without constraint firstly. Then the paths that exceed the passenger flow line constraint and guidance information constraint are selected for dynamic adjustment, which means that increasing the distance of that section makes the distance of passenger flows through that section increase, thus allowing passengers to choose another path to meet the passenger line and guidance information constraint. After the distance matrix is updated, the shortest path without constraint is calculated again for the passenger flow passing through these sections. The above operation is repeated until the algorithm finds a feasible solution. In the algorithm, $\alpha$ and $\beta$ are the weight factors for adjusting the path distance. The larger the weight factor is, the faster the algorithm converges but the accuracy cannot be guaranteed. The smaller the weight factor is, the closer it is to the optimal solution but the slower it converges. The specific steps of the heuristic algorithm are designed as shown below:

Step 1: Set the set of all passenger flows as the set of adjusted passenger flows. Turn to Step 2;

Step 2: Calculate the shortest path set Shortest_set of adjusted passenger flows without the constraint of passenger flow line and the constraint of the number of node guidance information. Turn to Step 3;

Step 3: Calculate the sum of the current passenger flow lines $W_{i j}$ between each two nodes and the sum of the number of guidance information $B_{i}$ at each node in the road network. Turn to Step 3;

Step 4: When the total number of passenger flow lines between each two nodes $W_{i j}$ exceeds the maximum passenger flow line limit $M a x_{-} W_{i j}$, adjust the distance of the road between these two nodes $D_{i j}=D_{i j}+\alpha W_{i j} / M a x_{-} W_{i j}$. Otherwise turn to Step 4;

Step 5: When the total number of guidance information of node $i$ exceeds the maximum number of allowed information $M a x \_B_{i j}$, adjust the distance of all roads connecting to this node $D_{i j}=D_{i j}+\beta B_{i j} / M a x_{-} B_{i j}$. Otherwise turn to Step 5;

Step 6: Find the passenger flow of all adjusted roads in the set of the shortest path without constraint Shortest_set and update it to the set of adjusted passenger flows. The shortest path of the passenger flow that has not adjusted the path and the node is put into the best shortest set Best_Shortest_set;

Step 7: if the adjusted passenger flow is an empty set, then output the optimal solution the best shortest path set Best_Shortest_set. Otherwise turn to Step 2.

\subsection{Instance verification}

Xizhimen station is one of the three three-line transfer stations of Beijing rail transit, where Beijing subway line 2, Beijing subway line 4 and Beijing subway line 13, and it is also the western terminal of line 13. Xizhimen station is the most central transfer hub station of Beijing rail transit, and it has the characteristics of complex spatial structure, large passenger flow, many transfer modes, many transfer paths with duplication, etc. Since Xizhimen station faces a large passenger flow pressure and many passenger flow lines, using Xizhimen station as a validation object has both scientific research value and strong practical significance.

In order to carry out the instance verification, the alternative locations of the rail transit signs are firstly determined. In this study, the alternative points for the sign placement are selected at the intersection of the passing roads, the up and down elevator entrance and the entrance/exit of the Xizhimen station. 
Next, verify the data source for passenger flow. Station congestion has always been an important challenge for rail transit stations. During the peak commuting period, the huge inbound, outbound and transfer passenger flow can easily cause station congestion, leading to low passage efficiency. Therefore, this study focuses on the effectiveness of station sign in guiding passenger flow during peak periods. During the morning peak, the commuting time is more concentrated, so the passenger flow during morning peak tends to be larger than that during evening peak. Therefore, this study uses passenger flow data from 7:00-9:00 a.m. during morning peak at Xizhimen Station. The data for the study were obtained from the passenger flow data of Beijing Rail Transit from December 25, 2018 to December 31, 2018. The data from 7:00-9:00 a.m. at Xizhimen Station each day were screened using Python software and then averaged to obtain the passenger flow at Xizhimen Station during the peak commuting period, as shown in Table I.

Table I: Passenger flow in Xizhimen station.

\begin{tabular}{|c|c|c|c|c|c|c|c|c|c|}
\hline \multirow{2}{*}{$\begin{array}{c}\text { Passenger flow } \\
(\text { person/h) }\end{array}$} & \multicolumn{5}{|c|}{ Entrance and exit } & $\begin{array}{c}\text { Station of } \\
\text { line 2 }\end{array}$ & $\begin{array}{c}\text { Station of } \\
\text { line 4 }\end{array}$ & $\begin{array}{c}\text { Station of } \\
\text { line 13 }\end{array}$ \\
\cline { 2 - 10 } & & A1 & A2 & B & C & D & T & F & D \\
\hline \multirow{4}{*}{ Entrance and exit } & A1 & - & - & - & - & - & 992 & 264 & 140 \\
\cline { 2 - 10 } & A2 & - & - & - & - & - & 992 & 264 & 140 \\
\cline { 2 - 10 } & B & - & - & - & - & - & 992 & 264 & 140 \\
\cline { 2 - 10 } & C & - & - & - & - & - & 992 & 264 & 140 \\
\cline { 2 - 9 } & D & - & - & - & - & - & 992 & 264 & 140 \\
\hline Station of line 2 & T & 1792 & 1828 & 1344 & 1344 & 1832 & - & 10192 & 20856 \\
\hline Station of line 4 & F & 688 & 688 & 688 & 688 & 688 & 15280 & - & 4784 \\
\hline Station of line 13 & D & 556 & 556 & 556 & 556 & 556 & 24400 & 8128 & - \\
\hline
\end{tabular}

Finally, we should guarantee that the flow line chosen by each passenger is within a certain volume of traffic, and set a reasonable number of guidance signs. If the amount of information is messy, it will cause interference to passengers when receiving information; if the amount of information is little, it cannot effectively guide passengers to their destinations.

\section{SIMULATION AND OPTIMIZATION ANALYSIS}

\subsection{Simulation model}

Anylogic pedestrian repository is a pedestrian simulation and crowd analysis tool that allows users to accurately model, visualize and analyse crowd behaviour in the physical environment and eliminate possible inefficient behaviour. This section still takes Beijing Xizhimen station as an example, and constructs a simulation model for rail transit station sign acquisition. Xizhimen station faces a large passenger flow pressure, especially in the area of Line 2 and Line 4. The area is shown in Fig. 1. Each initial place or destination has a target line, and each path sets a path. Target line indicates the target line as the starting position and destination position of pedestrians. Path indicates the path, which is used to specify the walking route of pedestrians. If a pedestrian enters from exit A to take Line 2, his starting position is exit A, following path, path 1 , path 2 , path 3 , path 28 , and finally arriving at Line 2 . If the pedestrian enters from exit A to take Line 4, his starting position is exit A, following path, path 4, path 5, and finally arriving at Line 4. 


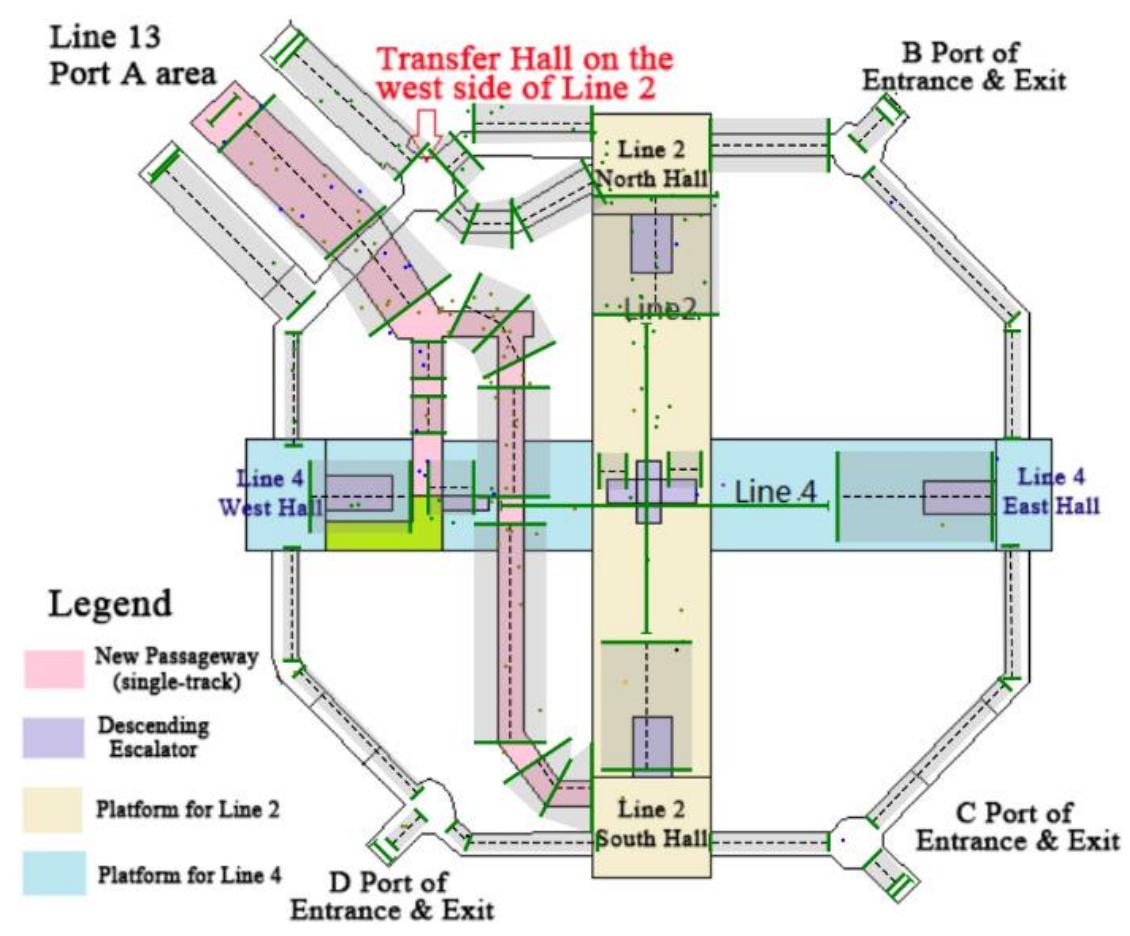

Figure 1: Line 2 to Line 4 simulation area in Xizhimen station.

Before using Anylogic software to obtain simulations of rail transit station signs from Line 2 to Line 4 in Xizhimen station, the following assumptions need to be made in order to reflect the real situation.

Assumption 1: The referenced pedestrian speed is set to obey a uniform distribution in the interval of $(4,6) \mathrm{km} / \mathrm{h}$ without external influence.

Assumption 2: The actual size of the Xizhimen station and the size of the rail transit station in the figure. Determine the coordinate scale of $3.52 \mathrm{pixel} / \mathrm{meter}$.

Assumption 3: The number of pedestrians in Beijing's rail transit has a strong relationship with time, and this paper uses the number of pedestrians in the morning peak as well as the weekend for analysis. The number of transfer pedestrians is assumed to be $60 \%$ of the number of outbound pedestrians.

Assumption 4: Through fieldwork, the research team marked Xizhimen station sign. Green cells indicate outbound signs, red cells indicate inbound signs, and yellow cells indicate both inbound and outbound signs.

Assumption 5: The spatial structure and sign types of Xizhimen station are divided into seven categories. The probability of acquiring the sign refers to the probability of passengers seeing and understanding the sign. The probability of each flow direction indicates the probability of passengers going to that flow direction when they do not acquire the sign.

\subsection{Simulation result analysis}

The operational efficiency of rail stations can be measured by indicators such as the average number of stranded passengers and passenger entrance and exit times. In this section, two indicators, the average number of stranded passengers and passenger entrance and exit times, are used for the analysis, and the time point for the data statistics was from December 25, 2018 to December 31, 2019.

(1) Analysis of simulation results of benchmark model on weekdays

The average number of stranded passengers in the station (the number of passengers in the passage) is 627 for the weekday morning peak period when the statistics are collected and the simulation model is run for one hour. The specific distribution of passengers' entrance and exit 
time is shown in Fig. 2. Average 163.76 seconds for transferring from Line 4 to Line 2 (North Hall), average 164.24 seconds for transferring from Line 4 to Line 2 (South Hall), average 76.06 seconds for entering Line 2 (North Hall) and average148.04 seconds for entering Line 2 (South Hall). Average165.5 seconds for transferring from Line 2 to Line 4 (West Hall), average 180.36 seconds for transferring from Line 2 to Line 4 (East Hall), average 37.65 seconds for transferring from Line 2 to Line 4 (elevator), average 92.47 seconds for entering Line 4 (West Hall), and average 104.6 seconds for entering Line 4 (East Hall).

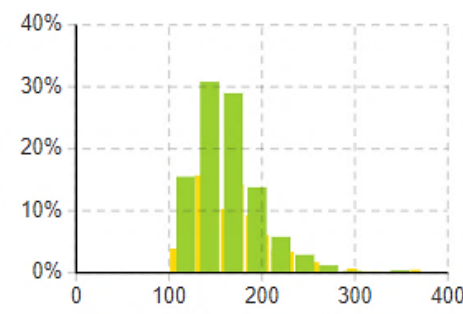

Line4 To Line2(North Hall) Time Distribution 163.76

Line4 To Line2(South Hall) Time Distribution 164.24

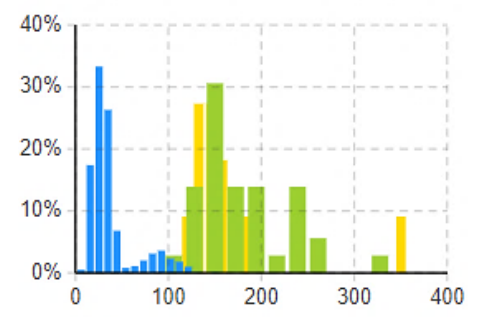

Line2 To Line4(West Hall) Time Distribution 164.6

Line2 To Line4(East Hall) Time Distribution 180.36

Line2 To Line4(Descending Escalator) Time Distribution 37.65

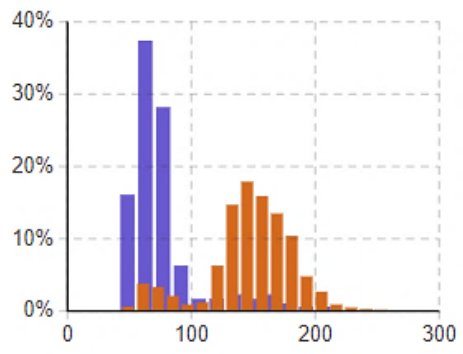

Line2(North Hall) Entrance Time Distribution 76.06

Line2(South Hall) Entrance Time Distribution 148.04

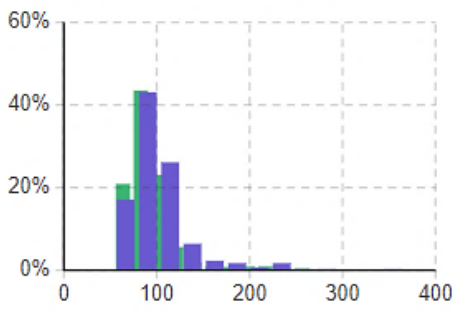

Line4(West Hall) Entrance Time Distribution 92.47 Line4(East Hall) Entrance Time Distribution 104.6

Figure 2: Time distribution of passengers entering and leaving the station (1).

(2) Analysis of simulation results of benchmark model during peak period

The average number of stranded passengers in the station (referring to the number of passengers in the passage) is 573 when running the simulation model for one hour, which is $8 \%$ less than the benchmark model. The specific distribution of passenger entrance and exit time is shown in Fig. 3. Average 187.31 seconds for transferring from Line 4 to Line 2 (North Hall), average 188.32 seconds for transferring from Line 4 to Line 2 (South Hall), average 84.09 seconds for entering Line 2 (North Hall) and average 107.41 seconds for entering Line 2 (South Hall). Nobody transfers from Line 2 to Line 4 (West Hall), average 205.29 seconds for transferring from Line 2 to Line 4 (East Hall), average 39.93 seconds for transferring from Line 2 to Line 4 (elevator), average 106.72 seconds for entering Line 4 (West Hall), and average 110.32 seconds for entering Line 4 (East Hall).

From the simulation results, the average number of stranded passengers in the station and the average travel time are higher during the morning peak hours than during weekdays. Although passengers travelling during the morning peak hours are more familiar with the routes, the average travel time is longer due to congestion at the rail stations caused by the excessive number of travellers. 

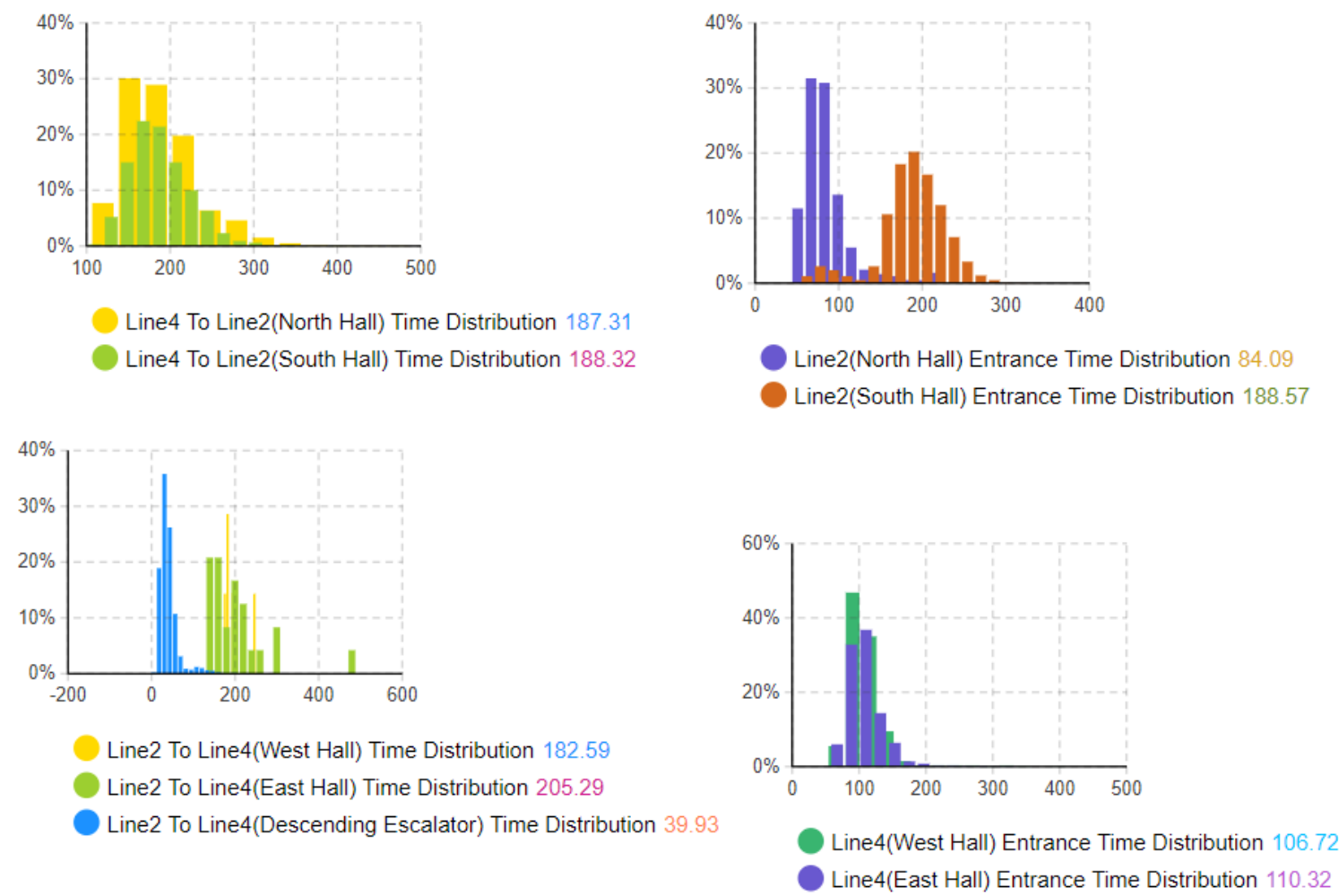

Figure 3: Time distribution of passengers entering and leaving the station (2).

\subsection{Optimal result analysis}

The areas with high passenger flow lines and density are the places where congestion occurs, so the probability of obtaining the sign of this area should be improved mainly. Using the Density Map component of the Anylogic pedestrian repository, the density of pedestrians in the simulation area can be tracked and identified by different colours. The project runs the model using the benchmark parameters of sign acquisition probability and records the heat map of passengers' activities, as shown in Fig. 4. The following diagram shows that the density of passengers is quite high on the station of Line 2, the station of Line 4, new passage at entrance A, and exit A1. The reason for the high density of passengers on stations of Line 2 and Line 4 is that all passengers need to enter the station of Line 2 or Line 4. And the reason for the high density of passengers on new passage at entrance $A$ is that there are many passengers entering the station from entrance A (including transferring from Line 13 to Line 2 or Line 4), especially from entrance A to Line 2. The reason for the high density of passengers in the exit A1 is that there are more passengers leaving the station from Line 2 to exit A1 (or transferring to Line 13). Therefore, both from the perspective of passenger flow lines and passenger density, it is important to increase the probability of obtaining signs in these areas.

According to the above analysis, priority should be given to optimizing the signs on the station of Line 2, the station of Line 4, the new passage of entrance A and the exit A1 passage area. The optimization strategy consists of two parts. Firstly, to increase the number of signs at the locations where there are already signs to improve the probability of obtaining signs; secondly, to set new signs at the locations where there were no signs before.

After optimizing the sign according to the above strategy, the weekday model is selected to run the simulation model, while the data indicators for leaving from exit A are added to collect relevant statistics. In order to facilitate the analysis of the results before and after the sign optimization, the indicators related to the sign optimization are selected for analysis. The average time to leave from exit A1 of Line 2 is 96.97 seconds, saving time compared with the benchmark model. The average entrance time of Line 2 (South Hall) is 136.85 seconds, saving 
nearly 10 seconds compared with the benchmark model. The average transfer time from Line 2 to Line 4 (elevator) is 26.78 seconds, saving more 10 seconds compared with the benchmark model. The average entrance time of Line 4 (West Hall) is 80.78 seconds, saving15 seconds compared with the benchmark model.

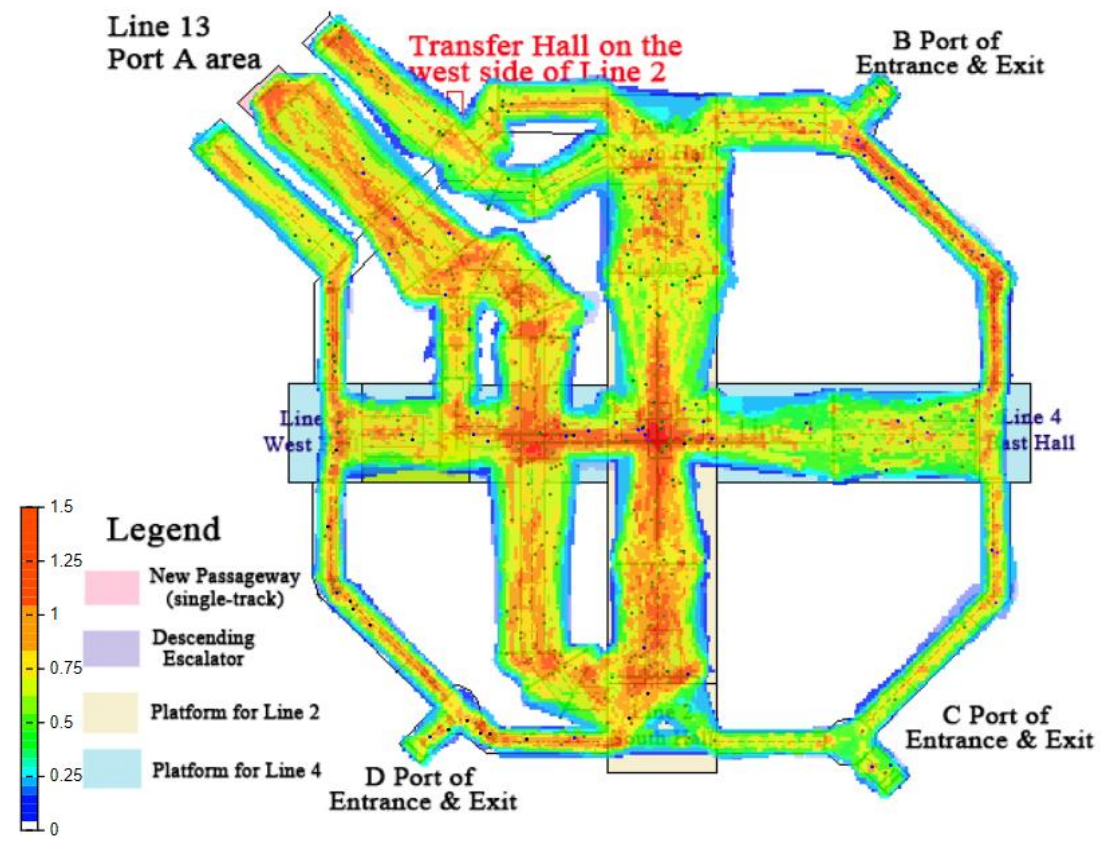

Figure 4: Passenger flow of Line 2 to Line 4.

In Fig. 5, it can be seen that these four passenger flow passages are leaving from exit A of Line 2 (including transferring from Line 2 to Line 13), taking Line 2 from entrance A (including transferring from Line 13 to Line 2), transferring from Line 2 to Line 4, and entering from entrance A to take Line 4 (including transferring from Line 13 to Line 4). These four have the practical significance of sign optimization because of the large passenger flow lines and passenger density.

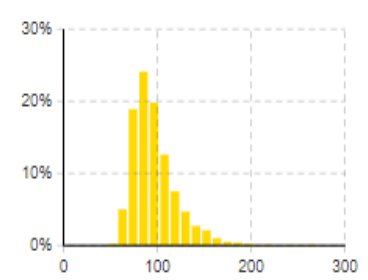

Line2 To Port-A1 Time Distribution 96.97 Line4 To Port-A1 Time Distribution

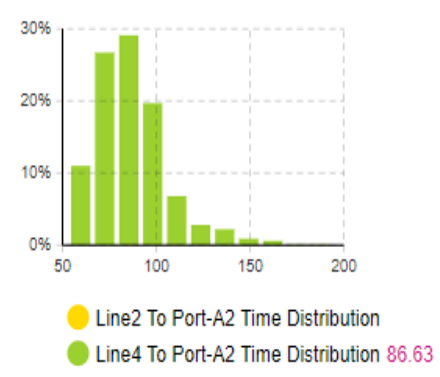

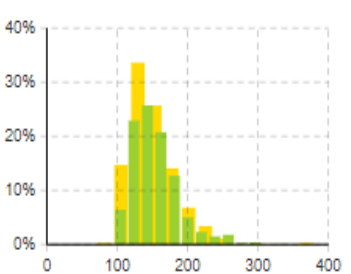

Line4 To Line2(North Hall) Time Distribution 148.85 Line4 To Line2(South Hall) Time Distribution 154.19

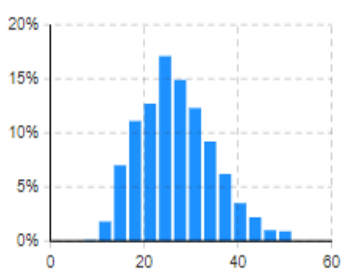

Line2 To Line4(West Hall) Time Distribution Line2 To Line4(East Hall) Time Distribution Line2 To Line4(Descending Escalator) Time Distribution 26.78

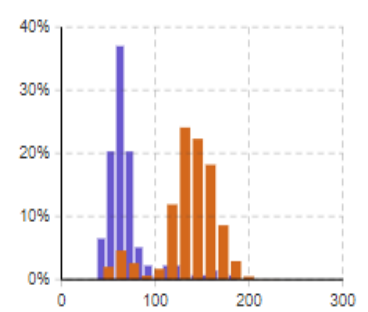

Line2(North Hall) Entrance Time Distribution 69.12 Line2(South Hall) Entrance Time Distribution 136.85

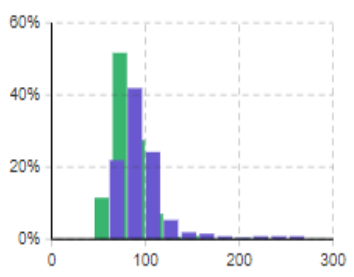

Line4(West Hall) Entrance Time Distribution 80.78 Line4(East Hall) Entrance Time Distribution 98.37

Figure 5: Optimized sign location map of Line 2 to Line 4. 
Fig. 6 shows the passenger flow density after sign optimization. By comparing with Fig. 5, it can be seen that the passenger flow density of station of Line 2, station of Line 4, the new passage of entrance $A$ and the passage of exit A1 have been reduced, indicating that this optimization strategy can effectively reduce the passenger flow density in the passage, enhance the capacity of the subway station and improve the passenger flow efficiency.

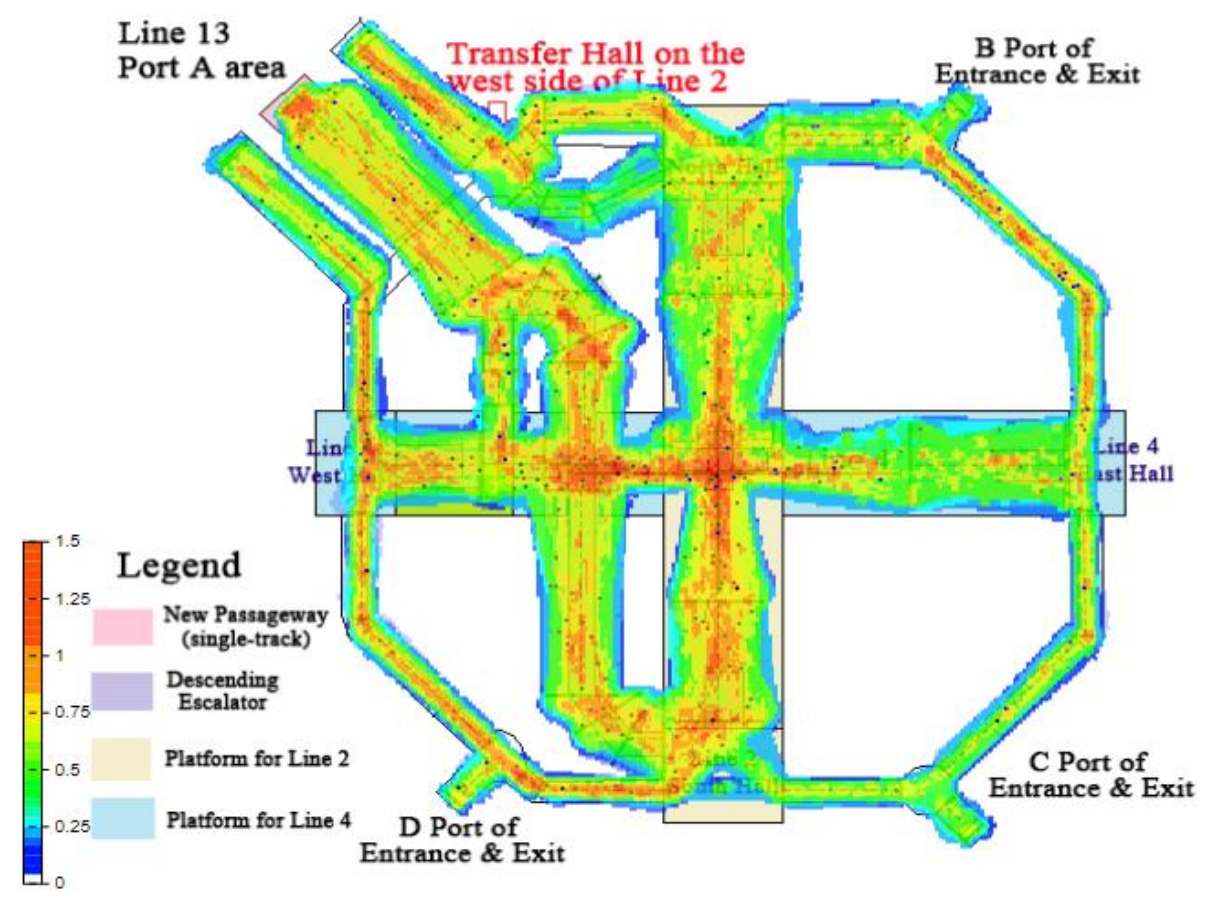

Figure 6: Optimized passenger flow of Line 2 to Line 4.

According to the research results, it can be known that the guidance signs for transferring between Line 2 and other public transportation at Xizhimen station are not perfect. Therefore, according to optimization model calculation results, wall attached type, ground type and induced type guidance signs can be set, which are beneficial for passengers to find the signs in the process of transferring. Setting and optimizing the guidance signs here can meet the needs of passengers for correct and smooth transferring and strengthen the role of rail transit management in guiding the flow of people. In addition, based on the importance of passenger flow, enhancing the guidance signs to the important core passenger location is another important goal of the optimization of guidance signs. The passenger flow line during transferring between Line 2 and Line 13 is larger than the transferring between other lines. Once the guidance signs cannot guide passengers to transfer in time, it will increase the waiting time of passengers and cause congestion problems. According to the optimization model results, the place that passengers must pass when they take Line 2 to Xizhimen station to get off and change to line 13 is also a dense intersection of passenger flow. In such an environment with a large flow of people, aerial hanging type, attached type and induced guidance signs should be set. Optimizing the setting of guidance signs at these four points can avoid the herding and blindness in the process of transferring and enhance the mobility of passengers.

The optimized alternative location of guidance signs suits the actual situation of Xizhimen Station, which not only allows passengers to find guidance signs within a certain range, but also reflects the continuity of the signs. Also, a certain number of signs can accurately provide passengers with guidance information, so that the amount of inducing information from guidance signs can be maximized. The model is effectively verified by real cases, avoiding the number of excessive and complex signs to confuse passengers' thinking and affect travel. The reasonable and scientific setting of guidance signs in such a complex location is necessary to 
guide passengers to travel smoothly. Therefore, the optimization model has realistic effectiveness, which greatly plays the important role of guidance signs and improves the efficiency of passenger transferring and rail transit operation.

\section{CONCLUSION}

Guidance signs are intended for passengers in that environment and are a medium for transmitting information to passengers. This study aims to enable passengers to reach their destinations in the shortest possible time. In order to enable passengers to reach their destinations as soon as possible, reasonable guidance signs can be set up so that passengers can pass through the shortest route, thus reducing the time passengers spend in the station and the number of passengers passing through the station at the same time.

The article transforms the problem of guiding sign placement into a mathematical optimization model, and establishes an optimization model for the placement of guiding signs in urban rail transit stations with the objective function of maximizing the amount of guidance information in the study area to help find the optimal placement of guiding signs scientifically from multiple alternative locations to reduce the guidance errors caused by guiding signs. The simulation selects Beijing rail transit Xizhimen station as an example for analysis, introduces the Xizhimen junction station, and analyses the passenger flow line of Xizhimen station. Based on this, the values of various parameters required for the practical application of the mathematical model were determined, so as to obtain a complete design model for the optimization of the layout of guide signs, and Anylogic software was chosen to solve the model. The research results can provide a basis for the practical application of sign optimization, and provide a reality check.

\section{ACKNOWLEDGEMENT}

This work was supported by the Fundamental Research Funds for the Central Universities (No. 3132021270).

\section{REFERENCES}

[1] Puttipakorn, P.; Upala, P. (2018). Comparative analysis of environmental graphic design for wayfinding on the exit patterns of mass transit stations, Open Transportation Journal, Vol. 12, 150-166, doi:10.2174/18744478018120100150

[2] Jiang, Z. B.; Gu, J. J.; Han, Y. Z.; Fan, W.; Chen, J. J. (2018). Modeling actual dwell time for rail transit using data analytics and support vector regression, Journal of Transportation Engineering, Part A: Systems, Vol. 144, No. 11, Paper 04018071, 12 pages, doi:10.1061/JTEPBS.0000189

[3] Yang, X. Y. (2013). How to get information in the process of way-finding, the research of the effectiveness of signage system design, Applied Mechanics and Materials, Vol. 437, 973-976, doi:10.4028/www.scientific.net/AMM.437.973

[4] Galea, E. R.; Xie, H.; Deere, S.; Cooney, D.; Filippidis, L. (2017). Evaluating the effectiveness of an improved active dynamic signage system using full scale evacuation trials, Fire Safety Journal, Vol. 91, 908-917, doi:10.1016/j.firesaf.2017.03.022

[5] Denis, J.; Pontille, D. (2014). Maintenance work and the performativity of urban inscriptions: the case of Paris subway signs, Environment and Planning D: Society and Space, Vol. 32, No. 3, 404416, doi:10.1068/d13007p

[6] Farhat, W.; Sghaier, S.; Faiedh, H.; Souani, C. (2019). Design of efficient embedded system for road sign recognition, Journal of Ambient Intelligence and Humanized Computing, Vol. 10, No. 2, 491-507, doi:10.1007/s12652-017-0673-3

[7] Zhang, L.; Liu, M.; Wu, X.; AbouRizk, S. M. (2016). Simulation-based route planning for pedestrian evacuation in metro stations: a case study, Automation in Construction, Vol. 71, Part 2, 430-442, doi:10.1016/j.autcon.2016.08.031 
[8] Guo, Z.; Wei, Z.; Wang, H. (2016). The expressway traffic sign information volume threshold and AGS position based on driving behaviour, Transportation Research Procedia, Vol. 14, 3801-3810, doi:10.1016/j.trpro.2016.05.465

[9] Upchurch, J.; Fisher, D.; Carpenter, R. (2002). Freeway guide sign design with driving simulator for Central Artery-Tunnel: Boston, Massachusetts, Transportation Research Record: Journal of the Transportation Research Board, Vol. 1801, No. 1, 9-17, doi:10.3141/1801-02

[10] Olander, J.; Ronchi, E.; Lovreglio, R.; Nilsson, D. (2017). Dissuasive exit signage for building fire evacuation, Applied Ergonomics, Vol. 59, Part A, 84-93, doi:10.1016/j.apergo.2016.08.029

[11] Jansen-Osmann, P.; Fuchs, P. (2006). Wayfinding behavior and spatial knowledge of adults and children in a virtual environment: the role of landmarks, Experimental Psychology, Vol. 53, No. 3, 171-181, doi:10.1027/1618-3169.53.3.171

[12] Wong, L. T.; Lo, K. C. (2007). Experimental study on visibility of exit signs in buildings, Building and Environment, Vol. 42, No. 4, 1836-1842, doi:10.1016/j.buildenv.2006.02.011

[13] Hund, A. M.; Padgitt, A. J. (2010). Direction giving and following in the service of wayfinding in a complex indoor environment, Journal of Environmental Psychology, Vol. 30, No. 4, 553-564, doi:10.1016/j.jenvp.2010.01.002

[14] Hund, A. M.; Minarik, J. L. (2006). Getting from here to there: spatial anxiety, wayfinding strategies, direction type, and wayfinding efficiency, Spatial Cognition \& Computation, Vol. 6, No. 3, 179-201, doi:10.1207/s15427633scc0603 1

[15] Vilar, E.; Rebelo, F.; Noriega, P. (2014). Indoor human wayfinding performance using vertical and horizontal signage in virtual reality, Human Factors and Ergonomics in Manufacturing \& Service Industries, Vol. 24, No. 6, 601-615, doi:10.1002/hfm.20503

[16] Basso, L. J.; Jara-Díaz, S. R. (2012). Integrating congestion pricing, transit subsidies and mode choice, Transportation Research Part A: Policy and Practice, Vol. 46, No. 6, 890-900, doi:10.1016/j.tra.2012.02.013

[17] Tirachini, A.; Hensher, D. A.; Rose, J. M. (2014). Multimodal pricing and optimal design of urban public transport: the interplay between traffic congestion and bus crowding, Transportation Research Part B: Methodological, Vol. 61, 33-54, doi:10.1016/j.trb.2014.01.003

[18] Istokovic, D.; Perinic, M.; Vlatkovic, M.; Brezocnik, M. (2020). Minimizing total production cost in a hybrid flow shop: a simulation-optimization approach, International Journal of Simulation Modelling, Vol. 19, No. 4, 559-570, doi:10.2507/IJSIMM19-4-525

[19] Buschiazzo, M.; Mula, J.; Campuzano-Bolarin, F. (2020). Simulation optimization for the inventory management of healthcare supplies, International Journal of Simulation Modelling, Vol. 19, No. 2, 255-266, doi:10.2507/IJSIMM19-2-514 